\title{
Helping high-risk families: Medical and public health approaches
}

Scientists, policy makers, and public health officials are beginning to explore how genetic knowledge can be used to promote health and prevent disease. One possibility for integrating human genetics into public health practice is to target disease prevention and health promotion efforts among individuals at high risk of disease because of their genetic makeup. Familial hypercholesterolemia $(\mathrm{FH})$ is a disease that is particularly well suited to this approach in that it is monogenic with a dominant pattern of inheritance, is relatively common in the U.S. population ( 1 in 500) when compared with other identified genetic conditions, is easily diagnosed, and is amenable to readily available treatments. The efficacy of early intervention and the cost-effectiveness of lipid-lowering drugs for persons with $\mathrm{FH}$ have also been established. In this issue, attributable fraction calculations by Austin and colleagues ${ }^{1}$ illustrate the potential public health impact of identifying and treating $\mathrm{FH}$ among relatives of persons with $\mathrm{FH} ; 44 \%$ of coronary heart disease (CHD) could be eliminated among male relatives, and $57 \%$ of CHD could be eliminated among female relatives. Austin and colleagues show that these percentages are higher than the $5 \%$ and $10 \%$ of CHD deaths (among males and females, respectively) that can be prevented in the general population through screening for hypercholesterolemia (assuming a 5\% prevalence in each group). However, we need to exercise caution in comparing these two sets of numbers. As the authors acknowledge, because of the low population prevalence of $\mathrm{FH}$, the identification and treatment of FH cases through familybased approaches would be unlikely to have a significant impact on CHD mortality in the general population. Because the pool of affected relatives is much smaller than the overall population, a $50 \%$ reduction in mortality among relatives may still lead to a lower number of overall prevented deaths in the population compared with a $5 \%$ reduction in mortality in the population at large.

Although the case for cascade testing is based on clinical and not public health considerations, case finding and interventions among relatives of $\mathrm{FH}$ cases should be encouraged. However, consensus has not been reached on the best methods for identifying persons with $\mathrm{FH}$, identifying their affected family members, and ensuring treatment and control of their cholesterol levels. Some persons with FH will be identified through routine blood cholesterol screening. In 2001, the National Cholesterol Education Program issued the Third Adult Treatment Panel (ATP III) report, which recommends screening for the U.S. population starting at age 20 and then repeated every 5 years. ${ }^{2}$ Over $70 \%$ of Americans have reported receiving cholesterol screening in the past 5 years. ${ }^{3}$ However, there are important geographic differences in screening rates and younger adults, men, and persons with no health insurance or persons who do not have a usual source of care are less likely to be screened than the general population. Potential FH candidates identified through cholesterol screening must undergo further evaluation to rule out secondary causes. Diagnosis of FH typically requires the presence of tendon xanthoma in either the candidate or a first- or second-degree relative, personal or family history of early CHD, or family history of elevated total or low-density lipoprotein (LDL) cholesterol, although the criteria for diagnosis varies across countries. Genetic testing is usually not feasible for diagnosis, because over 200 different mutations are associated with the syndrome. However, it may be useful in families with a known mutation or in areas where a founder effect and endogamy exist.

Targeted screening for identifying persons with $\mathrm{FH}$ has also been explored, including screening family members of patients with confirmed $\mathrm{FH}$ and evaluating patients with premature CHD (aged $<45-55$ years) and their families. Consensus has been reached by leading experts in the field that tracing $\mathrm{FH}$ through families is an efficient and cost-effective method of case ascertainment, ${ }^{4,5}$ and results by Austin and colleagues ${ }^{1}$ further support this conclusion. In addition, family screening may be more likely than other screening techniques to identify FH cases before they have overt disease, thereby enabling early intervention and lowering the patient's risk of experiencing an adverse cardiovascular event.

Although the ATP III report acknowledges the importance of addressing FH and screening family members, little guidance is given on how this should be accomplished. ${ }^{2}$ The roles of clinicians, health care organizations, insurers, health care benefit purchasers, and public health programs in screening and treating high-risk families-including those with $\mathrm{FH}$ - have not be adequately defined. Few physicians are screening close relatives of persons with abnormal lipids and positive family histories. For example, a review of outpatient medical records from the practices of 35 randomly selected primary care physicians in upstate New York found no mention of family screening in the records of patients with high cholesterol levels. ${ }^{5}$ The lack of testing and diagnosis of $\mathrm{FH}$ among family members was also observed in a survey of first- and seconddegree family members of persons with FH in Utah. Many family members had never been tested for cholesterol, and among those who reported a cholesterol level meeting the criteria for $\mathrm{FH}$, less than one third had been given a diagnosis of FH by their physician. ${ }^{5}$ Few studies have examined barriers to family screening by primary care physicians, but these barriers are likely to include lack of clinician expertise in the assessment and management of hyperlipidemia, reimbursement issues, 
privacy issues, the possibility of job and/or insurance discrimination, and the absence of a family-centered approach to health care. Many of these barriers also influence the provision of treatment. In the Utah study, only $42 \%$ of those with $\mathrm{FH}$ were on treatment and only $23 \%$ had cholesterol levels below the 90th percentile. ${ }^{5}$ These findings reflect inadequate treatment of high-risk patients in general; in 1999, only $45 \%$ of hospitalized patients with cardiovascular disease (CVD) who were receiving treatment had an LDL cholesterol level of $<130$ $\mathrm{mg} / \mathrm{dL}$ (which is above the ATP III goal of $<100 \mathrm{mg} / \mathrm{dL}$ ). ${ }^{3}$

Some communities have begun to create the infrastructure necessary for identifying and managing families with hyperlipidemia. Specific program components have included pooling health department and industry resources for $\mathrm{FH}$ program implementation, inviting physician input into program design, conducting physician and patient education on CVD risk factors and cholesterol treatment guidelines, providing access to lipid specialists in the patient's own community, and establishing new heart-health and lipid clinics. Initiatives that have been effective in increasing the treatment of other CVD risk factors are also likely to be effective in supporting the screening and treatment of high-risk families and include having health care benefit purchasers make family screening and treatment for elevated cholesterol a contractual obligation of insurers/providers, having health care organizations implement institutional changes (including a system for identifying persons with $\mathrm{FH}$ and their family members, automatically generated feedback on performance to promote provider intervention, dedicated staff for family screening and treatment follow-up, and reimbursement of clinicians and specialists for family screening), having screening and treatment of high-risk family members one of the standardized performance measures for health plans, and having health insurance companies and government medical programs cover the costs of patient and close relative identification and treatment. MedPed (Make Early Diagnosis-Prevent Early Deaths in MEDical PEDigrees) is an example of a program that has successfully used many of these methods for the past 13 years (see http://www.medped.org/). MedPed is currently being used by collaborators in over 30 countries to enhance diagnosis and treatment of persons with FH by registering them in the confidential MedPed database for patient education and follow-up and by making referrals to lipid specialists.

Although public health agencies and medical care systems are already supporting initiatives to improve the quality of care among persons at high risk of CVD by increasing the use of evidence-based treatment guidelines (i.e., the American Heart Association's Get With the Guidelines program [http://www. americanheart.org/] and the Health Resources and Services Administration's Diabetes and Cardiovascular Disease Collaborative [http://bphc.hrsa.gov/quality/collaboratives.htm]), these programs have not specifically targeted high-risk families. A recent meeting on genomics and chronic disease, conducted by the Chronic Disease Directors, a national organization affiliated with the Association of State and Territorial Health Officials, called for investigating the utility of targeting interventions to persons at high risk of chronic disease because of their family history of chronic diseases (see http://www.chronicdisease.org/Genomics_ Summit_Report.pdf). This emerging interest in using family history extends beyond finding rare high-risk families with monogenic disorders, to identifying the more frequent families at moderate risk of disease due to shared multiple genes and environmental factors and targeting behavioral and medical interventions. ${ }^{6}$

Persons at high risk deserve the highest priority in clinical intervention. For FH, identification and subsequent treatment can be best accomplished through family screening. Public health agencies, voluntary health organizations, and medical care providers responsible for CVD have an obligation to see that family screening is conducted, that persons with $\mathrm{FH}$ are provided with accurate diagnoses and appropriate counseling, and that they receive effective treatment.

\section{Ann M. Malarcher, PhD, MSPH Wayne H. Giles, MD, MS \\ Muin J. Khoury, MD, PhD \\ Centers for Disease Control and Prevention Atlanta, Georgia}

\section{References}

1. Austin MA, Zimmern RL, Humphries SE. High "population attributable fraction" for coronary heart disease mortality among relatives in monogenic familial hypercholesterolemia. Genet Med 2002;4:275-278.

2. National Cholesterol Education Program Expert Panel on Detection, Evaluation, and Treatment of High Blood Cholesterol in Adults. Executive summary of the third report of the National Cholesterol Education Program (NCEP) Expert Panel on Detection, Evaluation, and Treatment of High Blood Cholesterol in Adults (ATP III). JAMA 2001;285:2486-2497.

3. American Heart Association. 2002 heart and stroke statistical update. Dallas, Tex.: American Heart Association, 2001.

4. Marks D, Wonderling D, Thorogood M, Lambert H, Humphries SE, Neil HAW. Screening for hypercholesterolaemia versus case finding for familial hypercholesterolaemia: a systematic review and cost-effectiveness analysis. Health Technol Assess 2000;4:1-119.

5. Bild DE, Williams RR, Brewer HB, Herd JA, Pearson TA, Stein E. Identification and management of heterozygous familial hypercholesterolemia: summary and recommendations from an NHLBI workshop. Am J Cardiol. 1993;72:1D-5D.

6. Yoon PW, Scheuner MT, Peterson-Oehlke KL, Gwinn M, Faucett A, Khoury MJ. Can family history be used as a tool for public health and preventive medicine? Genet Med 2002;4:304-310. 